\title{
Register rerum ad Vol 14
}

(B) $=$ Buchbesprechung - Book review - Livre nouveau

$(\mathrm{R})=$ Referat - Review - Revue

Bearbeitet von G. Boehm, Basel

Ä-antigens, v. Antigens Agammaglobulinämie bei /?-Plasmozy-

tom, 250 Adrenalectomized rats, v. Inanition Agglutination, v. Leukaemia, studies

Agglutination, differential, v. Erythro-

cytes, survival time Agglutination test, v. Antigens Agranulocytose und Panmyelopathie,

327 (R) Agranulocytosis and leukocytopenia,

391 (R) Amniotic fluid, human, fibrinolyfic

system, 376 Amniotic fluid infusion, fibrinolytic activity in the circulating blood, 309 Anaemia, haemolytic, and haemolysis,

389 (R) Anaemia, mediterranean, v. Erythro-

cytes Anaemia, pernicious, 388 (R) Anaemia, pernicious, v. Intrinsic factor Anaemias, haemolytic, 336 (B) Anämie, akute, febrile, hämolytische, aregeneratorische, durch inkomplette Autoantikörper, 313 Anämie, aregeneratorische, v. Anämie, akute Anämie, febrile, v. Anämie, akute Anämie, hämolytische, v. Anämie, akute Animals; laboratory animals, handbook, 64 (B) Antibodies, multiple, incomplete, in normal human serum, 231 Antibodies, v. Autoantikörper, Leukaemia, studies Anticoagulants, 134 (R); 393 (R) Anticoagulants, spontaneous, $130(\mathrm{R})$ Antigen, v. Leukaemia, studies Antigens; presence of A- and B-antigens in human leukocytes examined by an agglutination test, 302 Antihaemophilic globulin (Factor VIII),

118 (R) Antikörper, v. Antibodies, Autoantikörper

Anti-platelet serum, v. Purpura Antithrombinaktivitäten des Plasmas,

ihre Bestimmung, 34 Aplasia of bone marrow, 390 (R) Autoantikörper, inkomplette; durch diese bedingte akute, febrile, hämolytische aregeneratorische Anämie, 313 -, v. Antibodies

B-antigens, v. Antigens

Biggs et Douglas, test, v. Thrombopla-stino-formatíon

Bilirubin and porphyrins, $387(\mathrm{R})$

Bleeding arrest; physiology, pharmacology, pathology, 64 (B)

Blood coagulation, physiology and pathology, review of the literature of 1954 (II.), $100 \mathrm{ff}$. (R)

Blood coagulation, v. Bleeding, Coagulation of blood

Blood groups and transfusions, $390(\mathrm{R})$

Blood groups, v. Antigens, Blutgruppen

Blood volume changes, v. Inanition

Blutbildungsstörungen, postfetale, v. Hämatopoese

Blutgruppen, $260(\mathrm{R})$

Blutgruppen, v. Antigens, Blood groups

Blutplasma, 257 (R) 
Blutthrombokinasebildung, v. Thrombo-kinase

Bone marrow aplasia, $390(\mathrm{R})$

Bone marrow, v. Calcium45, Knochen-marksuntersuchungen, Plasmazellen

Calcium (Factor IV), 116 (R) Calcium45, distribution in platelets and

bone marrow of rats, 291 Carbohydrates, v. Hydrates de carbone Castle; intrinsic factor, v.

Intrinsic factor Christmas factor (Factor IX), 119 (R) Coagulation of blood, v. Bleeding, Blood coagulation, Fibrin clot, Hydergine Colcemid, v. Leukaemia, myeloid,

chronic Colchicum derivative; treatment of chronic myeloid leukaemia, 83

396

Register rerum

XIV/1955

Congress, v. Société, Society

Cytology, v. Zytologie

Cytoplasmic nucleotides, v. Nucleotides

Demecolcin, v. Leukaemia, myeloid, chronic

Deutschsprachige hämatologische Lite-ratur im Jahre 1954 (unter Ausschluß der Blutgerinnung (I.), $257 \mathrm{ft}$; (II.), $321 \mathrm{ff}$. (R)

Dextran; its properties and use in medicine, 336 (B)

Diagnose (Differentialdiagnose), v. Enzyme

Diagnostik, klinische, Organpunktion, 270 (B)

Differential agglutination, v. Erythrocytes, survival time

Differentialdiagnose, v. Enzyme

Douglas et Biggs, test, v. Thromboplas-tino-formation

Eisen, v. Iron

Ematologia, attualità, 269 (B)

Embolie, v. Heparin

Embryo, v. Hämatopoese

Enzyme; differentialdiagnostischer Wert der Anwendung von Enzymen in der Hämatologie, 153

Ergot alcaloids, v. Hydergine

Erythroblasten, v. Hämatopoese

Erythroblastosen, fetale, $260(\mathrm{R})$

Erythrocytes; fever and heat as causes of fragmentation of red cells in patients with

mediterranean anaemia, 209

Erythrocytes and haemoglobin, 387 (R)

Erythrocytes, survival time; study of errors involved in the method of differential agglutination

for following transfused red cells, 88

Erythrocytes, v. Influenza virus

Erythropoese, $263(\mathrm{R})$

Factor I, 105 (R)

Factor II, 111 (R)

Factor III, 114 (R)

Factor IV, $116(\mathrm{R})$

Factor V (and VI), 116 (R)

Factor V, v. Faktor V

Factor VII, 117 (R) 
Factor VП-inhibitor; a new physiological serum accelerator inactivation principle, 57

Factor VII, v. Haemorrhagic diathesis

Factor VIII, 118 (R)

Factor IX, $119(\mathrm{R})$

Factor X, $120(\mathrm{R})$

Faim, v. Inanition

Faktor V, Einfluß auf die Plasma-Thromboplastinbildung, 294

Faktor V-Mangel, familiärer, Inhibiio-renstudien (II.), 65

-, v. Factor V

Fer, v. Iron

Ferments, v. Enzyme, Katalase

Fetus, v. Hämatopoese

Fever, v. Erythrocytes

Fibrin, 105 (R)

Fibrin clot and the prothrombin consumption, 22

Fibrinogen (Factor I), 105 (R)

Fibrinolysis, 105 (R)

Fibrinolytic activity in the circulating blood following amniotic fluid infusion, 309

Fibrinolytic system in human amniotic fluid, 376

Fragmentation of red cells, v. Erythrocytes

France; société française d'hématologie, seance special (réservée aux indications médicales des derives du sang), 272

Fucose, v. Hydrates de carbone

Fukose, v. Fucose

Galactose, v. Hydrates de carbone

Gamma-Globuline, v. Agammaglobu-linämie

Globulin, antihaemophilic(FactorVII), 118 (R)

y-Globuline, v. Agammaglobulinämie

Glucosamine, v. Hydrates de carbone

Glukosamin, v. Glucosamine

Granulopoese, 321 (R)

Guinea-pig, v. Influenza virus, Leukaemia, studies

Haematologie, v. Ematologia

Hämatologie (Differentialdiagnose), v. Enzyme

Haematology; international society of haematology; sixth congress, Boston, Mass., USA; 1956, 271

-, v. Société

Hämatopoese, embryonale, des Men-schen, Morphologie, im Vergleich zu postfetalen

Blutbildungsstörungen, 273

XIV/1955

Register rerum

397

-, v. Haemopoiesis Haemochromatosis, v. Haemosiderosís Haemoglobin and erythrocytes, 387 (R) Haemolysis and haemolytic anaemia, 
389 (R) Haemolytic serum, v. Influenza virus Haemophilia (general aspects), 121 (R)

Haemophilia; influence of Hydergine on the coagulation time in haemophilia, 96 Haemophilia, v. Antihaemophilic

globulin Haemopoiesis, v. Hämatopoese, Inanition Haemorrhagic diathesis due to a deficiency of factor VII (Hypoprocon-vertinaemia), 43 Haemorrhagic diathesis, v. Thrombo-

pathie Haemorrhagic disorders, 394 (R) Haemorrhagic disorders, v. Bleeding Hämorrhagische Diathese bei extremer Vermehrung der Thrombocyten (Thrombocythaemia haemorrhagica), 382 Haemosiderosis resembling haemochromatosis following multiple transfusions, 185 Heat, v. Erythrocytes Hématologie, v. Haematology Heparin and heparin-like substances, 133 (R) Heparin tolerance; limitations of heparin tolerance in the prediction of thromboembolism, 372 Heredopathie, v. Faktor V-Mangel,

Hodgkin's disease, Thrombopathie Hodgkin's disease, 392 (R) Hodgkin's disease, familial, 200 Hungerzustand, v. Inanition Hydergine; influence of hydergine on the coagulation time in haemophilia,96 Hydrates de carbone des paraprotéines sériques. Demonstration de la presence de fucose, galactose, mannose et glucosamine, 193 Hypoproconvertinaemia, v. Haemorrhagic diathesis

Immune-serum, v. Purpura

Inanition, effects upon haemopoiesis in the adrenalectomized rat with special reference to blood volume changes, 240

Influenza virus (PR 8 strain), adsorption on the red cells of guinea-pigs treated with haemolytic serum, 15

Inhibitorenstudien bei familiärem Faktor V-Mangel, 65

Intrinsic factor of Castle; a preliminary clinical trial of a purified preparation, 145

Iron and iron metabolism, $387(\mathrm{R})$

Isotope, radioaktíve, $\mathrm{v}$. Calcium 45

Japan, 207 (R)

Kaninchen, v. Thrombokinase Karger, Dr. Heinz, 60. Geburtstag, vor

209 Katalase, ihr Sinn, 238 Kern-Plasma-Relation von Plasmazel-

len des normalen Knochenmarks und

des multiplen Myeloms, 11 Kidneys, leukaemic, studies in their

enlargement, 354 Klinische Diagnostik, Organpunktion,

270 (B) Knochenmark, v. Bone marrow, Calcium45, Plasmazellen

Knochenmarksuntersuchungen, allge-

meine, 257 (R) Kohlehydrate, v. Hydrates de carbones

Laboratory animals, handbook, 64 (B)

Lapin, v. Thrombokinase

L.E. cells, $392(\mathrm{R})$

Leukaemia, 392 (R)

Leukaemia, myeloid, chronic, treatment with a Colchicum derivative, 83

Leukaemia, studies (I.). Aetiologic mechanism: Antigen-antibody techniques, 1

- (II.) Comparison of slide and test tube techniques for agglutination studies with guinea-pig serum, 7

Leukaemia, symposium (Kyoto), 207 (R)

Leukaemias, experimental, in the rat (Their development and transfer and some applications to the human problem), 337

Leukaemic kidneys, studies in their enlargement, 354 
Leukämie-Zellen, menschliche, Über-tragung auf Ratten, 181

Leukocytes, $391(\mathrm{R})$

Leukocytes, human, examined by an agglutination test, presence of A- and B-antigens, 302 398

Register

rerum

XIV/1955

Leukocytopenia and agranulocytosis,

$391 \quad(\mathrm{R})$

Leukopoese, v. Granulopoese Libri, 63 ff. (B); 269 (B); 336 (B) Lipidosis and reticuloendotheliosis, 393

(R)

Literatur, deutschsprachige, hämatolo-gische, im Jahre 1954 (unter Ausschluß der

Blutgerinnung) (I.), 257 fГ.; (II.), 321 ff. (R)

Literature, Scandinavian, on haemato-logy in 1954, review, 387 ff. (R)

Literature, v. Blood coagulation

Lupus erythematodes, v. L.E. cells

Lymphogranulomatosis, v. Hodgkin's disease

Man, v. Amniotic fluid, Antibodies, Antigens, Hämatopoese, Leukaemias, Leukämie-Zellen, Thrombokinase

Mannose, v. Hydrates de carbone

Megakaryocyten, v. Purpura

Megakaryocytes; cytoplasmic nucleotides in the megakaryocytes, 176

Metabolism, v. Iron

Methode, 257 (R)

Methode, v. Erythrocytes, survival time, Heparin, Laboratory animals, Leukaemia, studies

Myelom, multiples, v. Plasmazellen

Myeloma, multiple, and plasma cells,

$392 \quad(\mathrm{R})$

Myelome, multiple, v. Hydrates de car-bone

Nuclei, v. Kern-...

Nucleotides; cytoplasmic nucleotides in the megakaryocytes, 176

Organpunktion in der klinischen Dia-gnostik, 270 (B)

Panmyelopathie und Agranulocytose, 327 (R)

Paraprotein, v. Agammaglobulinämie

Paraprotéines sériques, v. Hydrates de carbone

PAS-staining, v. Hydrates de carbone

Plaquettes; affections plaquettaires, v. Thromboplastino-formation

Plasma, v. Blutplasma

Plasma (Zellplasma), v. Kern-Plasma-Relation

Plasma, Bestimmung seiner Antithrom-binaktivitäten, 34

Plasma cells and multiple myeloma, 392 (R)

Plasmaersatzstoffe, v. Dextran

Plasma thromboplastin antecedent (PTA), 120 (R)

Plasma-Thromboplastinbildung, Ein-fluß von Faktor V, 294 
Plasmazellen des normalen Knochen-markes und des multiplen Myeloms, Kern-Plasma-

Relation, 11

15-Plasmozytom, Agammaglobulinämie, 250

Platelets, $123(\mathrm{R})$

Platelets, v. Calcium45, Purpura, Throm-bopathie, Thromboplastino-formation

Polycythaemia vera, $390(\mathrm{R})$

Porphyrins and bilirubin, 387 (R)

Postfetale Blutbildungsstörungen, $v$. Hämatopoese

Proconvertin, v. Haemorrhagic diathesis

Proconvertin-Prothrombin, 393 (R)

Proteins, v. Agammaglobulinämie, Hydrates de carbone

Prothrombin (Factor II), 111 (R)

Prothrombin consumption and the fibrin clot, 22

Prothrombin-Proconvertin, 393 (R)

PTA, v. Plasma thromboplastin antecedent

PTC (Christmas factor), 119 (R)

Punktion, v. Organpunktion

Purpura, thrombocytopenic, induced by immune anti-platelet serum, experimental study, 72

Purpura, thrombocytopenische, experi-mentelle, Megakaryocyten und Thrombocytopoese, 215

Rabbit, v. Thrombokinase

Radiation injury, 390 (R)

Radioaktive Isotope, v. Calcium45

Rat, v. Calcium45, Inanition, Leukaemias, Leukämie-Zellen, Purpura

Red cells, v. Erythrocytes

Red cells, fragmentation, v. Erythrocytes

Relata, $100 \mathrm{ff}, 257 \mathrm{ff}, 321 \mathrm{ff}, 387 \mathrm{ff}$.

(R)

Ren, v. Kidneys

Reticuloendotheliosis and lipidosis, 393

(R)

Retikuläres System, 324 (R) Roesler; Robert Roesler de Villiers foundation, 63

XIV/1955

Register autorum

399

Scandinavian literature on haematology in 1954, review, $387 \mathrm{ff}$. (R)

Serum, human, v. Antibodies

Societates, 207 (R)

Société française d'hématologie, seance special (réservée aux indications mé-dicales des derives du sang), 272

Society, international of haematology, sixth congress, Boston, Mass., USA, 1956, 271

Strahlenwirkungen, v. Radiation

Stress, fourth annual report, 64 (B)

Survival time of erythrocytes, v. Ery-throcytes, survival time

Thalassaemia, v. Erythrocytes

Therapy, v. Colchicum derivative, Leukaemias 
Thoraxkrankheiten, klinische Zytologie, 63 (B)

Thrombin, $111(\mathrm{R})$

Thrombin, v. Antithrombinaktivitäten

Thrombinhemmung, v. Antithrombinaktivitäten

Thrombocyten, extreme Vermehrung, hämorrhagische Diathese (Thrombo-cythaemia

haemorrhagica), 382

Thrombocytes, 123 (R)

Thrombocytes, v. Calcium45, Purpura, Thrombopathie, Thromboplastino-formation

Thrombocythaemia haemorrhagica, 382

Thrombocytopenia, v. Thromboplas-tino-formation

Thrombocytopenic purpura induced by immune anti-platelet serum, experimental study, 72

Thrombocytopenische Purpura, experi-mentelle, v. Purpura

Thrombocytopoese, v. Purpura

Thrombo-embolism, v. Heparin

Thrombokinase; vergleichende Unter-suchungen über die Blutthrombo-kinasebildung bei

Kaninchen und Mensch, 363

Thrombopathia, v. Thromboplastino-formation

Thrombopathie hérédo-familiale mor-telle due à une insuffisance totale des thrombocytes, 202

Thromboplastin (Factor III), 114 (R)

Thromboplastin; plasma thromboplastin antecedent (PTA), 120 (R)

Thromboplastinbildung im Plasma, Ein-fluß von Faktor V, 294

Thromboplastino-formation dans les affections plaquettaires (Etude de 34 cas à l'aide du test de

Biggs et Douglas), 160

Thrombosis, $141(\mathrm{R})$

Transfusion, v. Erythrocytes, survival time

Transfusionen, $260(\mathrm{R})$

Transfusions and blood groups, 390 (R)

Transfusions, multiple, v. Haemosider-osis

Transfusionszwischenfälle, 260 (R)

Transplantation, heterologe, v. Leu-kämie-Zellen

Varia, 63, 271

Virus, v. Influenza virus

Vitamin B12, 388 (R)

Vitamin B12, v. Intrinsic factor

Vitamin K, 129 (R)

Zytologie, klinische der Thoraxkrankheiten, 63 (B)

-, v. Cyto. . .

Register autorum ad Vol. 14

$(B)=$ Buchbesprechung - Book review - Livre nouveau $(\mathrm{R})=$ Referat - Review - Revue

Ãkerman, L., v. Datta, N.J Albrechtsen, O. K., Storm, O., and

Trolle, D., 309 Albrechtsen, O. K., and Trolle, D., 376

Baserga, A., 269 (B)

Begemann, H., und Harwerth, H. G.,

257 ff., 321 ff. (R) Binswanger, D., Schaub, F., und Scheit-

lin, W., 382 
Braunsteiner, H., und Pakesch, F., 181 Brown, I. W. jr., v. Eadie, G. S. Bull, J. P., v. Squire, J. R. Camera, A., et Romagnoli, A., 202 Cheli, R., and Dianzani, M. U., 15 Curtis, W. G., v. Eadie, G. S. Dacie, J. V., 336 (B) Datta, N., Thorell, B., and Åkerman, L., 176 